\title{
Estudio de la fusión del asta mayor del hioides en relacion con la edad y revisión bibliográfica sobre las fracturas del hioides en medicina forense.
}

\author{
A study of the coalition of major cornua of the hioides in \\ connection with the age and bibliographical revision on the \\ fractures of the hioides in forensic medicine.
}

PM. Garamendi'1 , I. Alemán², MC. Botella² y MI. Landa ${ }^{3}$

\begin{abstract}
RESUMEN
Se presenta un estudio de revisión sobre las características generales de las fracturas del hioides en el contexto de la medicina forense. Junto con esta revisión, se incluye un estudio sobre el estado de fusión de las astas mayores con el cuerpo del hioides en una muestra de Ortopantomografías dentales digitales. La conclusión del trabajo es la de que el estado de fusión en la unión de las astas mayores del hioides con el cuerpo no puede predecirse por la edad o el sexo de un sujeto, ni tampoco puede presuponerse por este estado que vayan a existir o no fracturas de las astas mayores en casos de asfixia mecánica por compresión cervical. Se recomienda la práctica previa, en todos los casos de autopsias por asfixia mecánica con sospecha de compresión cervical de un estudio radiográfico del bloque cervical previo a la disección y, tras ésta, el análisis histopatológico del mismo para evitar errores de diagnóstico diferencial entre fracturas vitales y postmortales y ausencias de fusión del asta mayor con el cuerpo del hioides.
\end{abstract}

Palabras clave: Hioides; fractura; asfixia mecánica; autopsia médico forense; edad.

\section{Cuad Med Forense 2007; 13(50):227-242}

Trabajo parcialmente financiado por el Ministerio de Educación y Ciencia (ref. TIN2006-00829) y por la Consejería de Innovación, Ciencia y Empresa de la Junta de Andalucía (ref. TIC1619), ambos con fondos FEDER.

\section{ABSTRACT}

We present a review of the medico legal literature on hyoid bone fractures. It is also included a research on bone fusion of major cornua and hyoid bone body. This research has been conducted in a sample of digital dental Orthopantomographies. This research suggests that it isn't possible to predict the state of fusion of the major cornua in subjects of known sex and age. In cases of non union of major cornua it shouldn't be supossed that fractures of the hyoid won't happen. In all cases of forensic autopsies when mechanical asphyxia is suspected it is recommended to perform a radiological study of cervical tissues prior to extraction and after extraction it should be performed hystopathological studies.

Key words: Hyoid bone; fracture; mechanical asphyxia; forensic autopsy; age.

Correspondencia: Pedro Manuel Garamendi. IML de Huelva. Plaza Isabel La Católica, 9. 21071.Huelva. e-mail: imanolgaramendi@gmail.com.

1 Médico Forense. IML de Huelva.

2 Antropólogo Forense. Laboratorio de Antropología Física. Universidad de Granada.

3 Médico Forense. IVML. Subdirección de Vizcaya. 


\section{INTRODUCCION:}

Desde que en 1860 se describió por primera vez la fractura del hioides [I], este pequeño hueso del esqueleto laríngeo, pese a la infrecuencia general de su fractura $(0,002 \%$ del total de fracturas) ha sido objeto de especial estudio por parte de la Patología Forense. Las fracturas del hueso hioides se pueden encontrar en el cortejo de lesiones acompañantes a las asfixias mecánicas principalmente producidas por mecanismo de estrangulación manual. Dichas lesiones asientan fundamentalmente en sus astas mayores y en la producción de dichas fracturas el estado de fusión de la sincondrosis entre el asta mayor y cuerpo del hioides resulta de interés desde un punto de vista biomecánico. En el caso de que el asta mayor y el cuerpo se hallen fusionados, las fracturas pueden asentar en esta unión que parece constituir uno de los puntos débiles de la arquitectura del hioides ante compresiones laterales. Por otra parte, según algunos autores, el estado de no fusión de esta sincondrosis además podría actuar como un factor eventualmente protector frente a la producción de fracturas en el asta mayor del hioides. Cuando la unión no se ha llegado a osificar, la misma actuaría como un elemento elástico que disiparía las fuerzas de compresión que se ejercen sobre el hioides en la estrangulación manual y, por tanto, este estado de no fusión podría obligar a elevar el umbral de energía necesario para producir fracturas.

A pesar de su relativa importancia, son pocos los estudios que se han centrado en describir las características biomecánicas del hioides [2] o la forma en la que el estado de esta sincondrosis afecta a la posible producción de fracturas por compresión. También son escasos los estudios destinados a definir su ritmo de osificación con la edad y el sexo.

En el presente trabajo, se presentan las conclusiones de un estudio sobre una muestra de ortopantomografías (OPG) en las que se ha podido analizar el estado de fusión de 545 astas mayores del hioides en sujetos de ambos sexos y con amplia representación de edades. La ausencia de fusión del asta mayor puede ser una fuente de error en el diagnóstico diferencial de las fracturas del hioides. A la inversa, los estados de fusión pueden resultar facilitadores de la producción de fracturas de este hueso. Por ello, el conocimiento de si el estado de fusión del hioides es o no predecible a una determinada edad de los sujetos podría ayudar a interpretar correctamente posibles hallazgos compatibles con fracturas del hioides en sujetos de distintas edades. En caso contrario, sería necesario elaborar estrategias de diagnóstico destinadas a evitar errores diagnósticos en esta región anatómica.

\section{MATERIAL Y MÉTODOS:}

La muestra de estudio global ha estado constituida por 742 OPG digitales en formato JPG en las que, pese a la distorsión inducida por la técnica de obtención de las radiografías panorámicas dentales, se han podido identificar 323 OPG en las que se pudo valorar con suficiente nitidez las imágenes de uniones entre astas mayores y cuerpo del hioides. En 202 OPG ambas uniones han sido valorables. Las edades y sexo de los sujetos a los que se les practicaron las radiografías eran conocidos, aunque en el examen realizado no se identificaban estos datos.

Las radiografías se han analizado en formato digital obtenido mediante sistemas de elaboración de radiografía digital directa. Las imágenes radiográficas digitalizadas han sido facilitadas amablemente por un centro de radiodiagnóstico privado de Bilbao (Preteimagen) y la muestra de población de la que se han seleccionado las imágenes corresponde con una muestra aleatoria de sujetos estudiados en dicho centro. De esta manera, las radiografías han sido realizadas tanto a sujetos que presentan alteraciones patológicas en la región anatómica seleccionada (patología dental), como a sujetos sometidos a estas pruebas con carácter rutinario en pruebas de screening de población general. 
En las 323 OPG en las que se pudo identificar al menos una de las dos uniones, se ha analizado el aspecto morfológico de la unión entre asta mayor y cuerpo del hioides intentando definir en cada imagen si la misma correspondería con una unión completa, parcial o ausente. Para una adecuada visualización de las imágenes se han utilizado las funciones de alteraciones de brillo y contraste y de restricción de escalas de grises facilitadas por el programa Image J versión I.34 (versión más reciente 1.37). Este es un programa informático gratuito de código abierto que permite la visualización y el tratamiento de imágenes radiográficas digitales en distintos formatos, desde DICOM a JPG y que se halla disponible en versiones compatibles con plataformas Windows, Linux y Mac OS [3].

De este modo, en cada OPG se han registrado en cada uno de los dos lados del hioides si la unión presentaba uno de los siguientes valores: fusión completa, fusión parcial, fusión ausente o imagen distorsionada.

Por otra parte, se ha analizado también si en cada uno de los 202 hioides en los que ambas astas mayores eran identificables el estado de fusión de las mismas era unilateral, bilateral o ausente en ambas astas. Para los fines de esta estimación se ha considerado únicamente como fusión el estado completo de la misma para cada asta mayor del hioides.

Al tratarse de una serie en la que se comparan valores cualitativos (valores de fusión del asta mayor con el cuerpo del hioides) y cuantitativos (edad), los estadísticos analizados han incluido medidas estadísticas de distribución generales: media aritmética, desviación estándar, mediana, valores máximos y valores mínimos. Las medidas se han analizado por grupos de edad y por sexos. Gráficamente los resultados se han expresado mediante gráficos de cajas y bigotes (box-plot).

Se ha analizado la independencia de la selección de sexos en relación con los fenómenos de fusión mediante la prueba de Chi Cuadrado. Las diferencias con la edad para los parámetros de sexo y fusión se han analizado mediante análisis de varianza (ANOVA). La asociación de la edad con el grado de fusión en ambos sexos se ha cuantificado mediante el coeficiente correlación de Pearson considerando los tres posibles estados de fusión como fases de una variable cuantitativa.

Se incluyen ejemplos de cada uno de los valores definidos en las imágenes I y 2 . En la imagen 3, tomada de Papadopoulos et al en 1989, se ilustra una vista radiográfica de fusión unilateral del asta mayor del hioides [4].

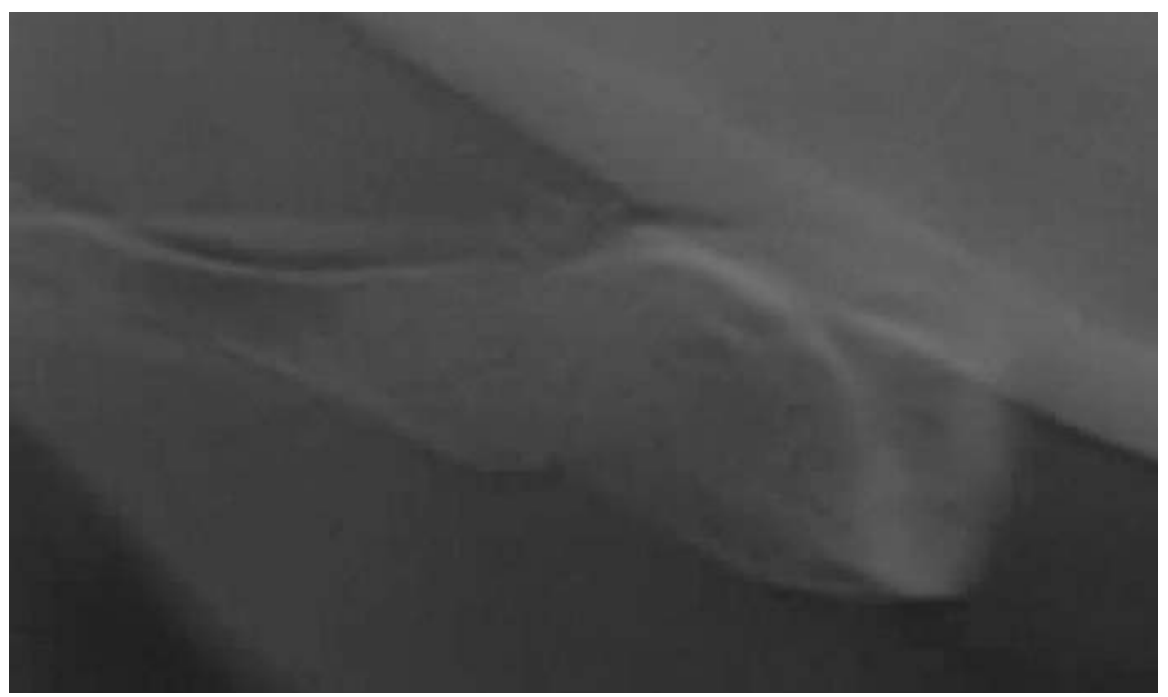

Imagen 1. Ortopantomografía dental. Fusión del asta mayor del hioides completa. 


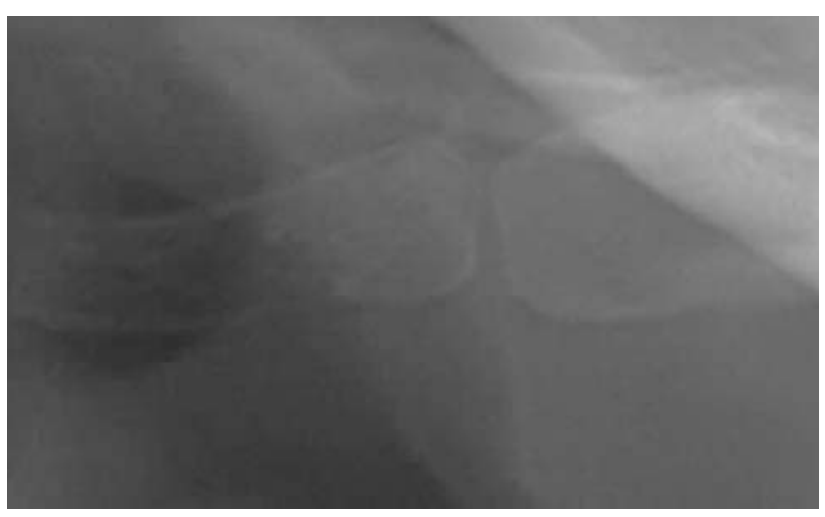

Imagen 2. Ortopantomografía dental. Fusión del asta mayor del hioides ausente.

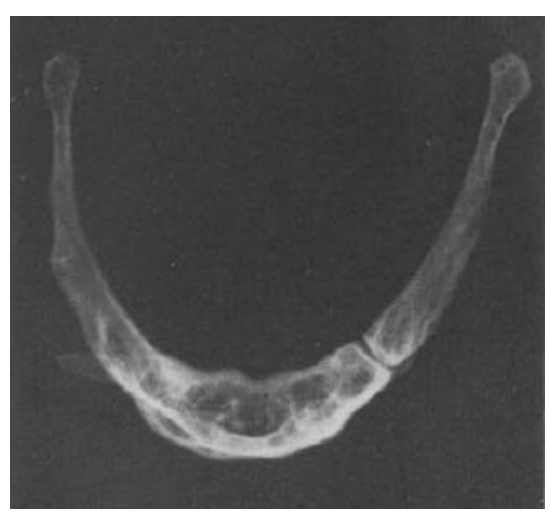

Imagen 3. Fusión unilateral del hioides en vista radiográfica superior. Tomada de Papadopoulos et al [29]

\section{RESULTADOS:}

La muestra de estudio original estuvo constituida por 742 OPG digitales. La distribución de la población original se recoge en la tabla I y en la gráfica I.

\begin{tabular}{|c|ccc|}
\hline Edad & Hombres & Mujeres & Total \\
\hline $0-20$ & 17 & 19 & 36 \\
$20-30$ & 58 & 63 & 121 \\
$30-40$ & 63 & 80 & 143 \\
$40-50$ & 74 & 110 & 184 \\
$50-60$ & 60 & 96 & 156 \\
$60-70$ & 40 & 35 & 75 \\
$>70$ & 12 & 15 & 27 \\
Total & 324 & 418 & 742 \\
\hline
\end{tabular}

Tabla 1. Ortopantomografías dentales por grupos de edad y sexo de la muestra global.

Las 323 OPG seleccionadas presentaron una distribución demográfica que se recoge en la tabla 2 y en la gráfica 2 . En estas OPG se han podido estudiar 545 uniones entre el asta mayor del hioides y el cuerpo. En 202 OPG, ambas uniones se pudieron identificar en la misma OPG.

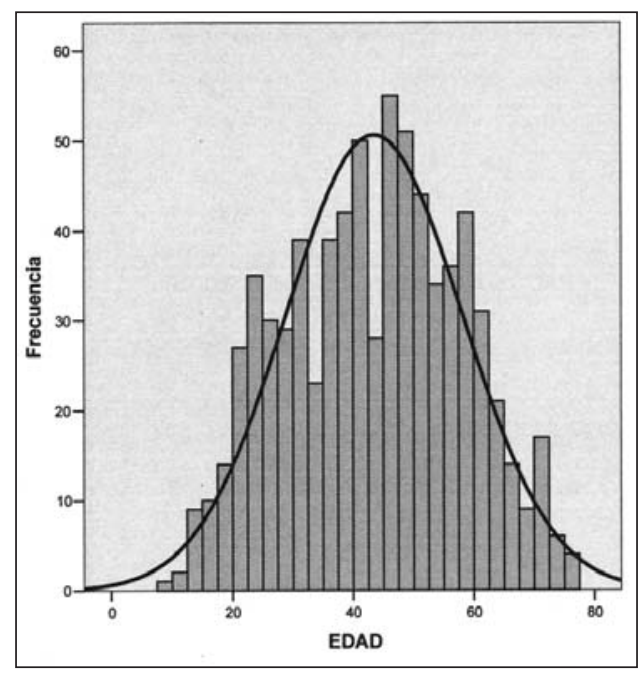

Gráfico 1. Histograma de frecuencias de edades para la muestra global de Ortopantomografías dentales.

Los resultados estadísticos para la fusión del asta mayor derecha e izquierda en la muestra general y de las muestras de hombres y mujeres se recogen en las tablas 3 y 4 y en los gráficos de cajas y bigotes (box-plot) 3 y 4 relativos a la muestra de ambos sexos.

Los resultados estadísticos para los fenómenos de fusión unilateral, bilateral y de ausencia de fusión para ambos sexos y para cada sexo se incluyen en las tablas 5, 6 y 7.

Los resultados más destacados de la serie analizada han sido:

- Para ambos sexos la edad media para los casos de no fusión se ha situado entre 33,58 y 33, 8 años de edad para ambas astas mayores, con un valor de desviación standard de 13,579 a |4,74 años en torno a la media. 
- Para ambos sexos la edad media para los casos de fusión completa se ha situado entre 44,9 y 45,34 años de edad para ambas astas mayores, con un valor de desviación standard de 12,877 a 13, 126 años en torno a la media.

- Los valores de las medianas de edad para casos de no fusión se han situado entre 30,77 y 31,9 años y para los casos de fusión completa entre 45,54 y 45,78 años en ambas astas mayores.

- Existen datos de fusión completa y bilateral de ambas astas mayores en sujetos de una edad mínima de 17 años de edad.

- Se aprecia una persistencia de imágenes de ausencia de fusión en ambas astas mayores en sujetos de hasta $6 \mathrm{I}$ años de edad. El número de casos en la muestra global con ausencia de fusión alcanza al 13,7\% de la muestra global, con una edad media de 32,79 años con una desviación standard de 14,653 años.

- Se aprecian datos de fusión unilateral en un 2,4\% de casos de la muestra global, con una edad media de 40,81 años y una desviación standard de 10,339. Los casos extremos máximos y mínimos para fusión unilateral correspondieron con sujetos de 52 y $2 \mathrm{I}$ años, respectivamente.

\begin{tabular}{|c|ccc|}
\hline Edad & Hombres & Mujeres & Total \\
\hline $0-20$ & 5 & 8 & 13 \\
$20-30$ & 22 & 29 & 51 \\
$30-40$ & 26 & 43 & 69 \\
$40-50$ & 29 & 60 & 89 \\
$50-60$ & 21 & 41 & 62 \\
$60-70$ & 17 & 10 & 27 \\
$>70$ & 7 & 5 & 12 \\
Total & 127 & 196 & 323 \\
\hline
\end{tabular}

Tabla 2. Distribución por grupos de edad y sexo de la muestra de OPG seleccionadas.

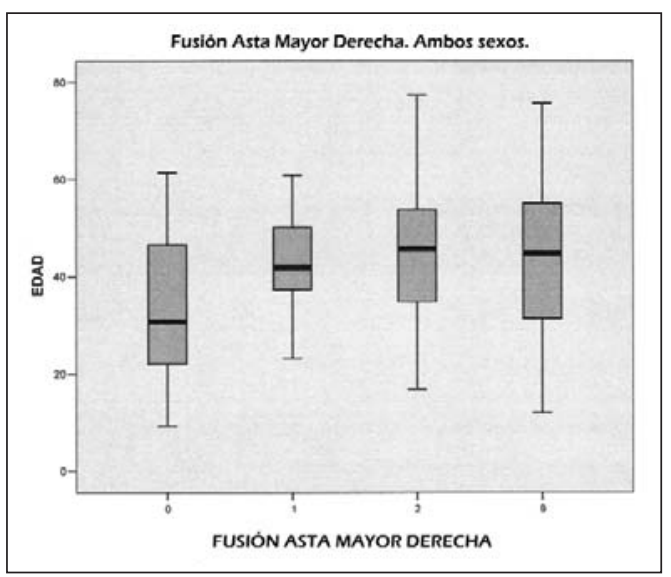

Gráfico 3. Diagrama de cajas de distribución de resultados de fusión, fusión parcial y no fusión en asta mayor derecha para ambos sexos (mediana, distancia intercuartil y rangos extremos). Valores de Fusión: 0 = No fusión; 1 = Fusión parcial; 2 = Fusión completa; 9 = Distorsionado o no visible.

Gráfico 4. Diagrama de cajas de distribución de resultados de fusión, fusión parcial y no fusión en asta mayor izquierda para ambos sexos (mediana, distancia intercuartil y rangos extremos). Valores de Fusión: 0 = No fusión; 1 = Fusión parcial; 2 = Fusión completa; 9 = Distorsionado o no visible.

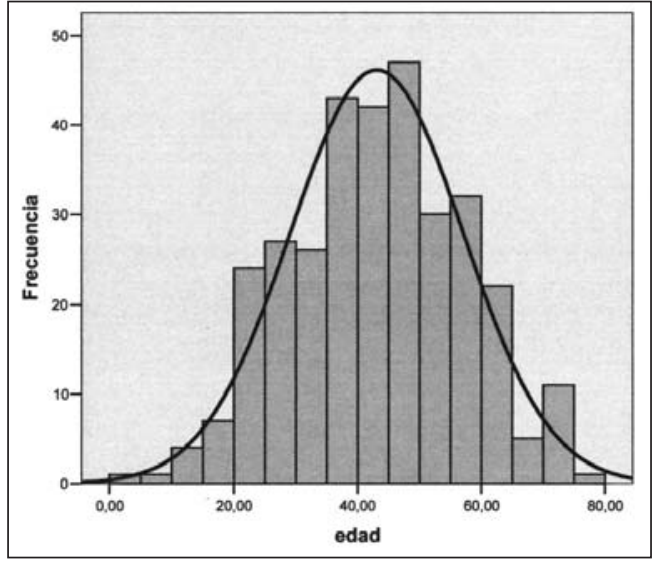

Gráfico 2. Histograma de frecuencias de edades para la muestra de 323 Ortopantomografías dentales seleccionadas.

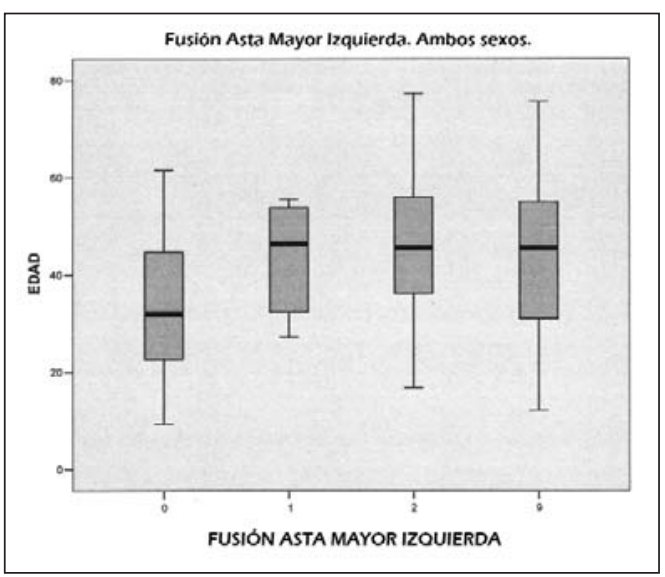




\begin{tabular}{|c|c|c|c|c|c|c|}
\hline \multicolumn{7}{|c|}{ Hombres } \\
\hline $\begin{array}{l}\text { FUSIÓN ASTA } \\
\text { MAYOR DCHA }\end{array}$ & Media & $\mathrm{N}$ & $\begin{array}{l}\text { Desviación } \\
\text { Standard }\end{array}$ & Minimo & Máximo & Mediana \\
\hline No Fusión & 35,30 & 11 & 17,910 & 9 & 61 & 38,89 \\
\hline Fusión Parcial & 52,68 & 4 & 10,299 & 38 & 61 & 56,11 \\
\hline Fus. Completa & 44,38 & 80 & 14,739 & 21 & 77 & 43,49 \\
\hline Perdidos & 43,02 & 229 & 15,182 & 12 & 76 & 43,63 \\
\hline Total & 43,21 & 324 & 15,177 & 9 & 77 & 43,47 \\
\hline \multicolumn{7}{|c|}{ Mujeres } \\
\hline $\begin{array}{l}\text { FUSIÓN ASTA } \\
\text { MAYOR DCHA }\end{array}$ & Media & $\mathrm{N}$ & $\begin{array}{c}\text { Desviación } \\
\text { Standard }\end{array}$ & Mínimo & Máximo & Mediana \\
\hline No Fusión & 32,83 & 25 & 13,422 & 14 & 57 & 30,08 \\
\hline Fusión Parcial & 39,38 & 15 & 9,978 & 23 & 52 & 40,92 \\
\hline Fus. Completa & 45,40 & 117 & 11,480 & 17 & 73 & 46,30 \\
\hline Perdidos & 44,28 & 261 & 15,094 & 14 & 74 & 45,61 \\
\hline Total & 43,73 & 418 & 14,185 & 14 & 74 & 45,26 \\
\hline \multicolumn{7}{|c|}{ Ambos sexos } \\
\hline $\begin{array}{l}\text { FUSIÓN ASTA } \\
\text { MAYOR DCHA } \\
\end{array}$ & Media & $\mathrm{N}$ & $\begin{array}{c}\text { Desviación } \\
\text { Standard } \\
\end{array}$ & Minimo & Máximo & Mediana \\
\hline No Fusión & 33,58 & 36 & 14,714 & 9 & 61 & 30,77 \\
\hline Fusión Parcial & 42,18 & 19 & 11,233 & 23 & 61 & 41,85 \\
\hline Fus. Completa & 44,99 & 197 & 12,877 & 17 & 77 & 45,78 \\
\hline Perdidos & 43,69 & 490 & 15,133 & 12 & 76 & 44,82 \\
\hline Total & 43,50 & 742 & 14,619 & 9 & 77 & 44,27 \\
\hline
\end{tabular}

Tabla 3. Medidas estadísticas de fusión, fusión parcial y no fusión del asta mayor derecha en hombres, mujeres y en ambos sexos

\begin{tabular}{|l|r|r|r|r|r|r|}
\hline \multicolumn{7}{|c|}{ Hombres } \\
\hline $\begin{array}{l}\text { FUSIÓN ASTA } \\
\text { MAYOR DCHA }\end{array}$ & \multicolumn{1}{c|}{ Media } & $\mathrm{N}$ & $\begin{array}{c}\text { Desviación } \\
\text { Standard }\end{array}$ & \multicolumn{1}{c|}{ Mínimo } & Máximo & Mediana \\
\hline No Fusión & 35,30 & 11 & 17,910 & 9 & 61 & 38,89 \\
Fusión Parcial & 52,68 & 4 & 10,299 & 38 & 61 & 56,11 \\
Fus. Completa & 44,38 & 80 & 14,739 & 21 & 77 & 43,49 \\
Perdidos & 43,02 & 229 & 15,182 & 12 & 76 & 43,63 \\
Total & 43,21 & 324 & 15,177 & 9 & 77 & 43,47 \\
\hline
\end{tabular}

\begin{tabular}{|l|r|r|r|r|r|r|}
\hline $\begin{array}{l}\text { FUSIÓN ASTA } \\
\text { MAYOR DCHA }\end{array}$ & \multicolumn{1}{|c|}{ Media } & N & $\begin{array}{c}\text { Desviacres } \\
\text { Standard }\end{array}$ & \multicolumn{1}{c|}{ Minimo } & Máximo & Mediana \\
\hline No Fusión & 32,83 & 25 & 13,422 & 14 & 57 & 30,08 \\
Fusión Parcial & 39,38 & 15 & 9,978 & 23 & 52 & 40,92 \\
Fus. Completa & 45,40 & 117 & 11,480 & 17 & 73 & 46,30 \\
Perdidos & 44,28 & 261 & 15,094 & 14 & 74 & 45,61 \\
Total & 43,73 & 418 & 14,185 & 14 & 74 & 45,26 \\
\hline
\end{tabular}

\begin{tabular}{|l|r|r|r|r|r|r|}
\hline FUSIÓN ASTA & \multicolumn{1}{|c|}{ Ambos sexos } \\
MAYOR DCHA & \multicolumn{1}{c|}{ Media } & N & $\begin{array}{c}\text { Desviación } \\
\text { Standard }\end{array}$ & \multicolumn{1}{c|}{ Minimo } & Máximo & Mediana \\
\hline No Fusión & 33,58 & 36 & 14,714 & 9 & 61 & 30,77 \\
Fusión Parcial & 42,18 & 19 & 11,233 & 23 & 61 & 41,85 \\
Fus. Completa & 44,99 & 197 & 12,877 & 17 & 77 & 45,78 \\
Perdidos & 43,69 & 490 & 15,133 & 12 & 76 & 44,82 \\
Total & 43,50 & 742 & 14,619 & 9 & 77 & 44,27 \\
\hline
\end{tabular}

Tabla 4. Medidas estadísticas de fusión, fusión parcial y no fusión del asta mayor izquierda en hombres, mujeres y en ambos sexos. 
Estudio de la fusión del asta mayor del hioides en relacion con la edad y revisión bibliográfica sobre las fracturas del hioides en medicina forense.

\begin{tabular}{|l|r|r|r|r|r|r|}
\hline $\begin{array}{l}\text { FUSIÓN } \\
\text { LATERALIDAD }\end{array}$ & \multicolumn{1}{c|}{ Media } & $\mathrm{N}$ & $\begin{array}{c}\text { Desviación } \\
\text { Estándar }\end{array}$ & \multicolumn{1}{|c|}{ Mínimo } & Máximo & Mediana \\
\hline No fusión bilateral & 32,79 & 27 & 14,653 & 9 & 61 & 31,46 \\
Fusión bilateral & 45,15 & 170 & 13,103 & 17 & 77 & 45,85 \\
$\begin{array}{l}\text { Fusión unilateral } \\
\text { derecha }\end{array}$ & 40,81 & 4 & 10,339 & 27 & 52 & 41,97 \\
$\begin{array}{l}\text { Fusión unilateral } \\
\text { izquierda }\end{array}$ & 21,38 & 1 & & 21 & 21 & 21,38 \\
\hline
\end{tabular}

Tabla 5. Distribución de resultados de la fusión según lateralidad en ambos sexos. Se incluyen sólo los casos de fusión completa.

\begin{tabular}{|l|r|r|r|r|r|r|}
\hline $\begin{array}{l}\text { FUSIÓN } \\
\text { LATERALIDAD }\end{array}$ & \multicolumn{1}{c|}{ Media } & N & $\begin{array}{c}\text { Desviación } \\
\text { Estándar }\end{array}$ & \multicolumn{1}{c|}{ Mínimo } & Máximo & Mediana \\
\hline No fusión bilateral & 37,11 & 10 & 17,783 & 9 & 61 & 39,18 \\
Fusión bilateral & 44,57 & 72 & 14,990 & 21 & 77 & 43,49 \\
$\begin{array}{l}\text { Fusión unilateral } \\
\text { derecha }\end{array}$ & 51,97 & 1 & & 52 & 52 & 51,97 \\
$\begin{array}{l}\text { Fusión unilateral } \\
\text { izquierda }\end{array}$ & $\cdot$ & $\cdot$ & $\cdot$ & $\cdot$ & $\cdot$ \\
\hline
\end{tabular}

Tabla 6. Distribución de resultados de la fusión según lateralidad en Hombres. Se incluyen sólo los casos de fusión completa.

\begin{tabular}{|l|r|r|r|r|r|r|}
\hline $\begin{array}{l}\text { FUSIÓN } \\
\text { LATERALIDAD }\end{array}$ & \multicolumn{1}{c|}{ Media } & $\mathrm{N}$ & $\begin{array}{c}\text { Desviación } \\
\text { Estándar }\end{array}$ & \multicolumn{1}{c|}{ Mínimo } & Máximo & Mediana \\
\hline No fusión bilateral & 30,25 & 17 & 12,349 & 14 & 49 & 24,09 \\
Fusión bilateral & 45,58 & 98 & 11,586 & 17 & 73 & 46,59 \\
$\begin{array}{l}\text { Fusión unilateral } \\
\text { derecha }\end{array}$ & 37,09 & 3 & 8,791 & 27 & 44 & 39,56 \\
$\begin{array}{l}\text { Fusión unilateral } \\
\text { izquierda }\end{array}$ & 21,38 & 1 & 21 & 21 & 21,38 \\
\hline
\end{tabular}

Tabla 7. Distribución de resultados de la fusión según lateralidad en Mujeres. Se incluyen sólo los casos de fusión completa.

La comparación de resultados de medias y desviaciones standard y medianas y distancias intercuartílicas tanto en las tablas aportadas como en los gráficos indican que la correlación con la edad del estado de fusión o no fusión no es muy significativa. Parece existir una diferencia entre las medias de edad a las que se observa el fenómeno de fusión o de no fusión, pero el solapamiento entre los rangos de edades definidos por las desviaciones standard y las distancias intercuartílicas en torno a la mediana hacen que esta diferencia sea poco significativa.

El análisis de los estadísticos descriptivos tampoco indica que exista una clara diferencia en 
el comportamiento entre ambos sexos para el fenómeno de fusión del asta mayor del hioides. La edad mínima a la que se observan datos de fusión completa en mujeres es de 17 años de edad frente a los $2 \mathrm{I}$ años de edad en el caso de varones. Sin embargo, la edad media a la que se alcanza el estado de fusión de las astas mayores en ambos sexos se sitúa en torno a los 45 años de edad, si bien exhibiendo ambos sexos desviaciones standard amplias con solapamiento de resultados y valores extremos que definen rangos de hasta 56 años para el estado de fusión completa.

La prueba de la Chi cuadrado para valorar la dependencia entre el sexo y el estado de fusión ha dado valores de 2,28 para el asta mayor izquierda (mínimo esperado con un nivel de significación del 5\%, 6,31) y de I,588 la izquierda (mínimo esperado con un nivel de significación del $5 \%, 2,76)$. Estos resultados indican la independencia del sexo para el estado de fusión en la muestra analizada.

El análisis de varianza ANOVA para la edad frente a sexo y estado de fusión se incluye en la tabla 8. Los valores F indican independencia de la edad frente al sexo y los estados de fusión en ambas astas mayores del hioides.

ANOVA

\begin{tabular}{|ll|r|r|r|r|r|}
\hline & & $\begin{array}{c}\text { Suma de } \\
\text { cuadrados }\end{array}$ & \multicolumn{1}{|c|}{ gl } & $\begin{array}{c}\text { Media } \\
\text { cuadrática }\end{array}$ & \multicolumn{1}{c|}{$\mathrm{F}$} & \multicolumn{1}{c|}{ Sig. } \\
\hline $\begin{array}{ll}\text { FUSIÓN ASTA } \\
\text { MAYOR DCHA. }\end{array}$ & Inter-grupos & 109,099 & 220 &, 496 &, 992 &, 633 \\
& Intra-grupos & 1,000 & 2 &, 500 & & \\
& Total & 110,099 & 222 & & & \\
& Inter-grupos & 52,774 & 220 &, 240 &, 960 &, 646 \\
& Intra-grupos &, 500 & 2 &, 250 & & \\
FEXXIÓN ASTA & Total & 53,274 & 222 & & & \\
MAYOR IZQDA. & Inter-grupos & 118,413 & 220 &, 538 &, 538 &, 842 \\
& Intra-grupos & 2,000 & 2 & 1,000 & & \\
& Total & 120,413 & 222 & & & \\
\hline
\end{tabular}

Tabla 8. Análisis ANOVA para edad frente a variables de sexo y fusión de ambas astas mayores del hioides (valor crítico $F$ $0,05(2,220)=3,04)$.

La correlación entre el estado de fusión del asta mayor del hioides, considerado como variable cuantitativa, y la edad arroja unos valores de coeficiente de correlación de Pearson de 0,295 para el asta derecha y 0,29I para la izquierda, ambos indicativos de una baja correlación.

\section{DISCUSIÓN:}

Embriogénicamente, el hueso hioides se desarrolla a partir de cartílagos del segundo y tercer arcos branquiales, el asta menor del segundo, el asta mayor del tercero y el cuerpo de la fusión de los extremos ventrales de ambos. En la quinta semana comienza su condrificación y ésta se completa en el tercer o cuarto mes intraútero [6]

Su osificación se produce a partir de 6 centros: dos para el cuerpo y uno para cada asta. Al principio, el cuerpo presenta una zona de osificación en banda que se ha descrito en niños a partir del $4^{\circ}$ mes de desarrollo intrauterino [6]. Las astas mayores presentan signos de osificación radioló- 
gica desde el $5^{\circ}[7]$ o $6^{\circ}$ mes de vida [6]. En el $75 \%$ de los recién nacidos hay signos de radiopacidad en el hioides [7].

Las astas menores se sitúan en el ángulo superior de la unión de cada asta mayor con el cuerpo (imagen 4). Son pequeñas eminencias cónicas que se unen mediante tejido fibroso al cuerpo y, ocasionalmente, mediante una articulación sinovial con las astas mayores. La osificación de estas astas es muy errática. Así, se ha podido observar tanto su ausencia de osificación, como su osificación completa fusionándose con el cuerpo. Las astas menores se unen también con el extremo hioideo del ligamento estilohiodeo, pudiendo existir calcificaciones de este ligamento que darían lugar a los huesos ceratohioideo y estilohiodeo. En sus formas más extremas, la osificación de la cadena estilohiodea puede ser completa, incluyendo los cartílagos de Reichter, timpanohioideo y estilohioideo y ceratohiodeo [8].

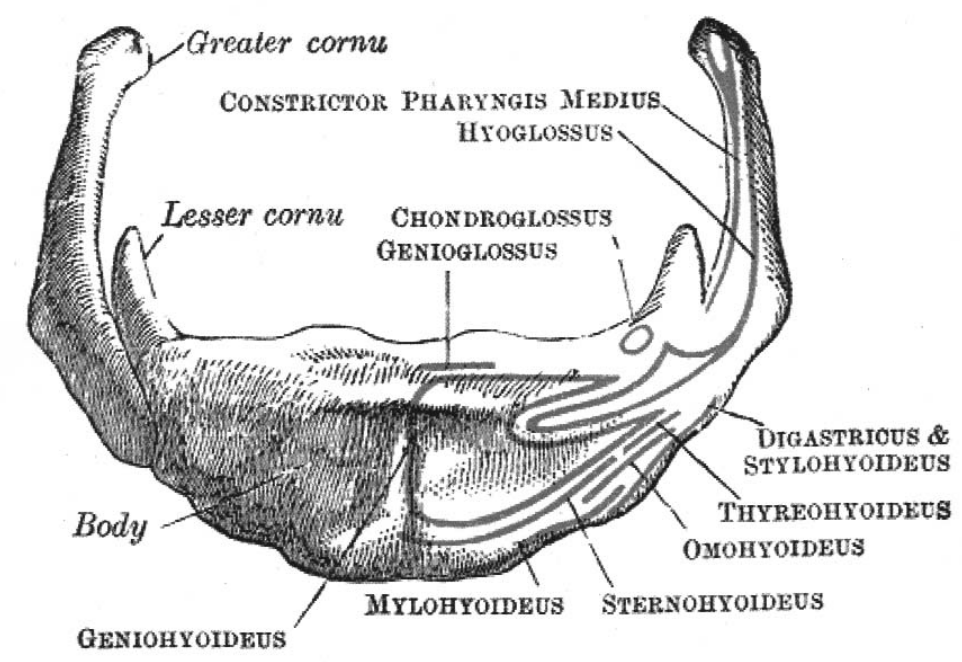

Imagen 4. Hioides. Vista anterior [5]

El proceso de fusión de las astas mayores con el cuerpo del hioides ha sido analizado en pocas series tanto desde el punto de vista osteológico como radiológico. Una de las pocas series previas es la analizada originalmente en 1987 por O'Halloran y Lundy [9] posteriormente ampliada por Miller, Walker y O'Halloran en 1998 [ [ 0]. La primera serie estuvo constituida por 300 hioides extraídos en autopsias, habiendo analizado los mismos en un tercio de casos sólo por observación osteológica directa y en los otros 200 casos además mediante métodos radiológicos. La serie ampliada estuvo constituida por 3 I 5 hioides en los que se utilizaron en todos los casos métodos radiológicos. La distribución de la segunda muestra incluyó I 88 hombres y 127 mujeres entre 2 meses y 98 años de edad. La proporción en la muestra total de varones con fusión unilateral fue del 19,7\%, bilateral del $31,9 \%$ y sin datos de fusión en el 48,4\%. En mujeres, hubo fusión unilateral en el $21,4 \%$, bilateral en el 34,9\% y sin datos de fusión en el 43,7\%. Los valores máximos de proporción de casos por grupo de edad de fusión unilateral en varones fueron del 34,5 \% en el grupo de 4 I a 50 años de edad y en mujeres en el grupo de 4 I a 50 años con una proporción del $31,8 \%$. Los valores máximos de proporción de casos por grupo de edad de fusión bilateral en varones fueron del $60 \%$ en el grupo de 7 I a 80 años de edad y en mujeres en el grupo de 7 I a 80 
años con una proporción del 52,2 \% [10]. En la tabla 9 se incluyen los resultados de la serie de O'Halloran y Lundy de 1987, en la que se observan tanto fenómenos de fusión como de no fusión en prácticamente todos los grupos de edad.

\begin{tabular}{lllll}
\hline Hombres & & & & \\
\hline Edad & No fusión & Fusión Unilateral & Fusión bilateral & Total \\
\hline $0-9$ & $3(100 \%)$ & 0 & 0 & 3 \\
$10-19$ & $22(100 \%)$ & 0 & 0 & 22 \\
$20-29$ & $34(69,4 \%)$ & $6(12,2 \%)$ & $9(18,4 \%)$ & 49 \\
$30-39$ & $20(40,0 \%)$ & $9(18,0 \%)$ & $21(42,0 \%)$ & 50 \\
$40-49$ & $11(27,5 \%)$ & $12(30,0 \%)$ & $17(42,5 \%)$ & 40 \\
$50-59$ & $11(35,5 \%)$ & $3(9,7 \%)$ & $17(54,8 \%)$ & 31 \\
$60-69$ & $2(14,3 \%)$ & $2(14,3 \%)$ & $10(71,4 \%)$ & 14 \\
$>70$ & $4(25,0 \%)$ & $2(12,5 \%)$ & $10(62,5 \%)$ & 16 \\
Total & $107(47,6 \%)$ & $34(15,1 \%)$ & $84(37,3 \%)$ & 225 \\
\hline Mujeres & & & & \\
\hline Edad & & & & \\
\hline $0-9$ & No fusión & Fusión Unilateral & Fusión bilateral & Total \\
\hline $10-19$ & $3(100 \%)$ & 0 & 0 & 3 \\
$20-29$ & $3(75 \%)$ & $1(25,0 \%)$ & 0 & 4 \\
$30-39$ & $10(58,8 \%)$ & $4(23,5 \%)$ & $3(17.6 \%)$ & 17 \\
$40-49$ & $3(23,1 \%)$ & $8(61,5 \%)$ & $2(15,4 \%)$ & 13 \\
$50-59$ & $5(38,5 \%)$ & $6(46,1 \%)$ & $2(15,4 \%)$ & 13 \\
$60-69$ & $3(33,3 \%)$ & $2(22,2 \%)$ & $4(44,4 \%)$ & 9 \\
$>70$ & $2(22,4 \%)$ & $5(55,6 \%)$ & $2(22,2 \%)$ & 9 \\
Total & $2(28,7 \%)$ & $1(14,3 \%)$ & $4(57,0 \%)$ & 7 \\
\hline & $31 \%)$ & $27(36,0 \%)$ & $17 \%)$ & 75 \\
\hline
\end{tabular}

Tabla 9. Resultados serie de O'Halloran y Lundy (1987) sobre fusión de astas mayores y cuerpo del hioides [9].

Otros autores, como Evans y Knight en 198I indicaron que se habían podido identificar casos de fusión completa en sujetos de apenas 18 años de edad y que, por el contrario, pudieron identificar casos de ausencia de fusión en sujetos de edades superiores a 80 años de edad [6].

Los tratados clásicos de Patología Forense, pese a la importancia que otorgan a la fractura 
del hioides en el contexto del diagnóstico patológico de la asfixia mecánica por estrangulación manual, no profundizan en exceso en definir en qué momento resulta previsible que el asta mayor se halle fusionada con el cuerpo del hioides. Así, el tratado de Casas y Rodríguez [I I] sólo indica que la osificación se produce en la edad media de la vida. El tratado de Patología Forense de Di Maio y Di Maio [12] no aporta datos sobre el momento de osificación del asta mayor del hioides con el cuerpo, al igual que el tratado de Simpson [13]. Del mismo modo, el clásico de Gisbert [14] tampoco aporta datos sobre este aspecto. El tratado de Knight simplemente indica que la fusión del asta mayor con el cuerpo del hioides sigue una secuencia errática, aunque es posible que en sujetos jóvenes la unión sea cartilaginosa y móvil y a partir de la edad media de la vida se encuentre calcificada y sea frágil $[15,16]$. Sin embargo, todos ellos hacen hincapié en la importancia del hallazgo de fracturas en este hueso en los casos de asfixia mecánica, especialmente por estrangulación manual.

Casas y Rodríguez indican que en los casos de estrangulación manual, las fracturas asientan en el extremo distal de las astas mayores a I cm de su extremo. Especifican que estas fracturas son excepcionales en sujetos jóvenes ya que las astas mayores no se hallarían fusionadas con el cuerpo. Indican, además, que este tipo de fractura puede observarse también en casos de ahorcadura y estrangulación a lazo [I I ]. Gisbert especifica que en los casos de estrangulación a lazo no se observan fracturas en el hioides, aunque sí en el cartílago tiroides. Este mismo autor no indica si esta diferencia se repite en las estrangulaciones manuales o en las ahorcaduras, aunque señala que las lesiones osteocartilaginosas son habituales en estos tipos de mecanismos de asfixia mecánica [14]. Di Maio y Di Maio indican que en su experiencia habían hallado fracturas del hioides en un $18 \%$ de los casos de estrangulación manual, mientras Simpson y Knight en 1985 habían reportado una proporción del 4,5\% [13]. En una serie posterior, Vincent Di Maio indica que pudo identificar casos de fracturas de hioides solas o asociadas a otras fracturas laríngeas en 28 casos de estrangulación manual. Describe fracturas aisladas del hioides en 9 casos [17]. En los casos de ahorcadura, Di Maio y Di Maio no identifican casos de fractura del hioides. Señalan en su tratado de 1993 que la fractura del hioides también es posible en casos de estrangulación a lazo, aunque se encuentra con menos frecuencia que la del tiroides [12]. El tratado de Simpson señala que es posible hallar fracturas del hioides en casos de estrangulación manual, pero con menor frecuencia que fracturas del tiroides [13]. La serie del INTCF de Madrid, publicada por Sánchez de León en 200 I, indicaba que en los casos de estrangulación manual la fractura de hioides se encontró en un $26 \%$ de casos, en los casos de estrangulación a lazo no se apreció ninguna fractura del hioides y en los casos de ahorcaduras se apreció fractura del complejo hioides-tiroides en un 44\% de los casos [ [ 8]. El tratado de Knight es el único que dedica un apartado específico al estudio más exhaustivo de las fracturas laríngeas en asfixias mecánicas. Este autor destaca que las fracturas del hioides son posibles incluso en sujetos jóvenes a pesar de que la sincondrosis del asta mayor pueda ser aún cartilaginosa. Del mismo modo, destaca la posibilidad de que las fracturas se produzcan en el contexto no sólo de estrangulaciones manuales sino también de ahorcaduras y de otros tipos de traumatismos cervicales o incluso postmortalmente. Recomienda que durante la disección se seccione cuidadosamente la membrana tirohioidea para evitar que un posible fragmento libre de fractura pierda su relación con el resto del hioides. Recomienda, asimismo, la práctica del estudio radiográfico del bloque cervical previa a la disección $[15,16]$.

Felgin indicaba en 1999 que de 307 casos de ahorcadura pudo identificar fracturas de hioides en 10 casos [19]. Del mismo modo, Betz y Eisenmenger en 1996 identificaron fracturas de tiroides e hioides en un 67\% de su serie de 109 muertes por ahorcadura, destacando casos de fracturas en sujetos de edades tan tempranas como los 14 años [20]. 
Pese a la aparente falta de acuerdo entre las series sobre la modalidad de asfixia mecánica en la que se puede apreciar la existencia de fracturas de hioides, todas ellas coinciden en indicar que la estrangulación manual es la modalidad en la que resulta más previsible la aparición de fracturas del hioides y en la que su aparición es más significativa como hallazgo patológico para poder establecer el diagnóstico de la modalidad de asfixia mecánica. Sin embargo, a pesar de la infrecuencia relativa de este tipo de fracturas, debe tenerse siempre en mente en la interpretación de los resultados en casos de autopsias por este posible mecanismo de muerte que las fracturas del hioides no han sido descritas únicamente en casos de estrangulación.

Así, fuera del contexto de las asfixias mecánicas, la fractura del hioides ha sido descrita tanto aislada como en asociación con fracturas de la mandíbula. Estas últimas han sido fundamentalmente descritas en casos de accidentes de tráfico por atropello, especialmente en aquellos casos en los que tras el impacto inicial existe arrollamiento secundario del atropellado, siendo común su asociación con fracturas de otros elementos del esqueleto laríngeo $[1,21]$. Antes del advenimiento de los sistemas de retención en vehículos a motor la fractura de hioides se describía también en conductores cuyo cuello impactaba contra el volante. Existen múltiples referencias de fracturas aisladas provocadas por mecanismo de hiperextensión cervical brusca, por degluciones bruscas del bolo alimenticio [I] o por el stress inducido por el vómito [22]. Pese a que en algunas series no se han podido identificar fracturas del hioides provocadas por la intubación orotraqueal (IOT) en maniobras de reanimación cardio-pulmonar (RCP) [23], otros autores sí que han identificado casos de producción de fracturas de hioides en el curso de maniobras de RCP por IOT e hiperextensión cervical inadecuada para liberación de la vía aérea [24]. Las fracturas del hioides se han descrito también en casos de heridas punzantes por arma blanca tanto mortales como con supervivencia. En la estadística del Instituto Vasco de Medicina Legal se cuenta un caso de fractura de hioides provocada por una herida cervical punzante por arma blanca autoinfringida con supervivencia del lesionado. Finalmente, en relación con el origen de las fracturas del hioides, ya autores como Gordon et al en 1976 alertaban sobre la posibilidad de provocar iatrogénicamente fracturas postmortales del hioides en el curso de las maniobras de autopsia al realizar la extracción del bloque cervical [25].

Las fracturas del hioides no se asocian necesariamente con cuadros clínicos severos que pongan en riesgo la vida del lesionado o que precisen asistencia médica urgente. Las manifestaciones clínicas de una fractura aislada del hioides pueden ser anodinas o debutar como cuadros de disfagia aguda severa con o sin laceración faríngea $[26,27,28]$ o de auténticas obstrucciones respiratorias agudas [29]. En estos casos, la inflamación y hemorragia reactivas a la fractura pueden llegar a provocar un efecto de masa en la faringe que produzca manifestaciones clínicas más o menos severas.

En sus series Maxeiner [30] y Khokhlov [3 I], ambos en 1999, indicaban que en casos de autopsias médico legales fue posible identificar fracturas antiguas consolidadas en el hioides. En su serie, Maxeiner indicaba que en un total de 1160 autopsias judiciales pudo identificar 12 casos de fracturas antiguas del hioides. Estos casos correspondieron en su mayoría con sujetos afectos de alcoholismo crónico. Khokhlov en una serie sobre 440 bloques cervicales identificó 7 casos ( I,6\%) de fracturas antiguas del hioides, en 5 casos localizadas en el extremo más distal del asta mayor y en I caso en la unión del asta mayor con el cuerpo. Todos ellos se describieron en sujetos de edades superiores a 35 años y raramente por encima de los 50 años. En este grupo de edad entre 35 y 50 años, la frecuencia en hombres duplicaba a la de mujeres. También Khokhlov sugirió la posible relación entre estas fracturas antiguas consolidadas con el alcoholismo en población urbana. Ambos autores sugirieron que posibles caídas casuales en estado de embriaguez pudieron haber sido en muchos casos la causa de las fracturas y la tendencia a no solicitar asistencia médica la razón de que no hubiesen sido diagnosticadas en vida de los fallecidos. 
Se ha sugerido que la morfología del hioides puede ser un factor predisponente para la producción de fracturas del hioides en casos de compresión por estrangulación manual. Así, autores como Koebke y Saternus en 1979 [32], Papadopoulos et al en 1989 [4] o Harjeet y Jit en 1996 [33] han sugerido que pueden clasificarse los hioides en función de su morfología en varios subtipos, que básicamente pueden ser divididos en dos grandes grupos (hiperbólicos y parabólicos) y sus variantes. En distintos estudios se han relacionado estos morfotipos con el sexo con hallazgos diversos. Sin embargo, Pollanen y Ubelaker en 1997 analizaron las características métricas de 100 hioides de los dos grupos morfológicos principales (hiperbólicos y parabólicos) y llegaron a la conclusión de que las diferencias en las medidas tomadas en ambos tipos de hioides eran poco importantes y no significativas. No se pudo apreciar un predominio de ningún morfotipo o medida en ningún grupo de edad y sexo y sólo pudo destacarse que las mujeres tendían a tener hioides con medidas más pequeñas que los hombres. En cuanto a una posible predisposición a la fractura en los hioides con astas mayores más largas, tampoco pudieron confirmar tal suposición propuesta por autores previos [34].

La localización de las fracturas del hioides en casos de estrangulación ha sido estudiada por Pollanen et al en 1995 [35]. Estos autores identificaron fracturas en el tercio distal del hioides en un $47 \%$ de casos ( 9 casos), en el tercio medio en un $47 \%$ de casos (9 casos) y en el tercio proximal en 5\% ( I caso). Algunos autores, como Casas y Rodríguez han sugerido que la ausencia de fusión del asta mayor del hioides con el cuerpo determinaría que no se produjesen fracturas del asta mayor del hioides en casos de estrangulación [ I I]. Sin embargo, tanto en la serie de Pollanen et al de 1995 [35] como en la de Pollanen y Chiasson de 1996 [36] se han podido identificar casos de coexistencia de fenómenos de no fusión del asta mayor del cuerpo del hioides y de fracturas del asta mayor en su tercio distal.

El mecanismo de producción de las fracturas del hioides en los casos de asfixia mecánica ha sido analizado biomecánicamente por unos pocos autores. En los casos de estrangulación Di Maio y Di Maio indican que es la compresión simultánea de ambas astas mayores por ambos laterales del cuello la que produce la fractura de una o ambas astas mayores [12]. Sin embargo, en estudios biomecánicos sobre resistencia y desplazamiento del hioides ante distintos tipos de fuerzas compresivas se ha podido comprobar cómo las cargas aplicadas directamente sobre el cuerpo del hioides en dirección antero-posterior pueden dar lugar a fracturas de las astas mayores, localizadas habitualmente en el tercio proximal y en la zona de la sincondrosis con el cuerpo. En un estudio publicado por Kasprzak et al en 1993 se sugería que esta modalidad de carga puede producir fracturas cuando las astas mayores quedan fijadas por la compresión contra la columna cervical y, de forma análoga a como ya señalaban Saternus y Koebke en 1979, sugieren que la dirección de la carga puede ser un factor determinante para que la fractura del hioides se produzca en unas $u$ otras zonas de las astas mayores, explicando de este modo en parte la diversa localización de las fracturas en las distintas series publicadas [2].

En su conjunto todos los datos bibliográficos parecen sugerir, como ya habían apuntado previamente Ubelaker y Pollanen [1,34], que en la producción de las fracturas del asta mayor del hioides en casos de estrangulación y de otras formas de asfixia mecánica provocadas por compresión cervical, la morfología del hioides o su estado de fusión o no fusión del asta mayor con el cuerpo no son datos determinantes para que se produzcan o no fracturas del asta mayor. Por el contrario, factores externos como la intensidad de la presión ejercida, la dirección en la que se ejerce la compresión o la propia posición del cuello en el momento de la compresión serían factores mucho más importantes en tanto determinantes de que se produzca o no la fractura del hioides y que ésta asiente en una $u$ otra zona del asta mayor. 
En la investigación médico forense de los casos de asfixia mecánica con sospecha de posible lesión ósea del hioides o del esqueleto laríngeo, todos los autores coinciden en recomendar que el estudio patológico no se base únicamente en un mero examen macroscópico de los bloques cervicales. Existe un elevado riesgo de interpretar erróneamente estados de no fusión del asta mayor con fracturas o de atribuir erróneamente un origen traumático a fracturas provocadas por la manipulación del bloque cervical durante su extracción. Por ello, recomiendan que en todos los casos de este tipo de autopsias tras la extracción del bloque cervical se analicen las piezas mediante métodos radiográicos e histopatológicos, con el fin de localizar y diferenciar correctamente auténticas fracturas vitales de fracturas postmortales o meras ausencias de fusión de la sincondrosis del asta mayor. Algunos autores, además, recomiendan el examen de los hioides mediante estereomicroscopio para identificar correctamente fracturas no desplazadas, lesiones en tejidos no osificados o fracturas antiguas $[15,16,18,37,38,39]$.

El análisis de la serie de OPG estudiadas, de forma análoga a lo citado por autores previos, indica que el fenómeno de fusión del asta mayor con el cuerpo del hioides no sigue una clara secuencia con la edad o el sexo. En la serie de OPG analizadas para este trabajo se han podido identificar casos de fusión completa a edades tan precoces como los 17 años de edad o casos de ausencia de fusión bilateral en sujetos con edades de hasta 61 años en esta serie o incluso edades superiores a 80 años en otras series $[6,10]$. Del mismo modo, en series previas se ha señalado que pueden apreciarse fenómenos de fusión unilateral en hasta un $20 \%$ de los sujetos estudiados [ 10$]$; en la serie que se ha analizado para este estudio esta proporción ha sido más escasa, de un $2,4 \%$ con una edad media de 40,8 años de edad y valores de edad extremos entre 21 y 52 años de edad. El análisis estadístico de las series analizadas, tanto la presente como otras precedentes, tampoco permite establecer que exista una comportamiento diferenciado de este fenómeno de fusión ósea con la edad en cada sexo considerado de forma independiente. En la serie de $\mathrm{O}^{\prime}$ Halloran y Lundy los casos de fusión unilateral más precoces se identificaron en mujeres de 18 años de edad y en hombres por encima de los 20 años de edad [9]. Este hallazgo de una aparente mayor precocidad del fenómeno de fusión del asta mayor del hioides también se ha reproducido en la serie analizada para este estudio, con una edad mínima de 17 años para fusión bilateral en mujeres frente a los 21 años en hombres, pero más allá de estos valores extremos mínimos el comportamiento en ambos sexos ha sido independiente y por igual poco predecible en relación con la edad.

Dado que, según se demuestra en esta serie de estudio y en series precedentes, el ritmo de fusión del asta mayor del hioides resulta errático en su relación con la edad, también es cierto que conocida la edad de un sujeto no será posible establecer con suficiente fiabilidad si el estado de fusión del asta mayor será completo, parcial o con ausencia de fusión a nivel uni o bilateral. De este modo, la existencia de una solución de continuidad en la unión del asta mayor del hioides en un adulto joven no debe ser considerada a priori como una sincondrosis no osificada, ya que no es improbable que se trate de una fractura y, a la inversa, en un sujeto en torno a los 60 años de edad toda solución de continuidad en la unión no puede ser considerada de antemano como una fractura ya que no puede descartarse que se trate de una sincondrosis no fusionada. Por ello, en los casos de autopsias por asfixias mecánicas con sospecha de lesión de este hueso la presencia de una imagen de solución de continuidad en el extremo proximal del asta mayor debe siempre ser evaluada con precaución, no debiendo presuponerse por la edad o el sexo de la víctima que la misma pudiera corresponder bien con una fractura o con un fenómeno de no fusión. Del mismo modo, por los datos de la revisión bibliográfica manejada no debe suponerse que un estado de no fusión del asta mayor sea incompatible con la existencia de otras fracturas del asta mayor del hioides, ya que están 
descritos casos de coexistencia de ambos hechos. Por todo ello y tal y como ya ha sido señalado anteriormente por otros autores, en los casos de sospecha de este tipo de lesión el examen de los bloques cervicales debiera incluir en todos los casos y de forma rutinaria un examen radiográfico previo a la disección de los tejidos y un examen histopatológico complementario para una adecuada interpretación de los hallazgos necrópsicos.

\section{AGRADECIMIENTOS:}

A los doctores Arsenio Martínez y Elena Lángara y a los técnicos del centro de radiodiagnóstico Preteimagen de Bilbao, sin cuya desinteresada colaboración facilitándonos la muestra de radiografías utilizada en el estudio este trabajo no hubiese sido posible.

\section{BIBLIOGRAFÍA:}

I. Ubelaker D. Hyoid fracture and strangulation. J Forensic Sci.1992;37(5):1216-1222.

2. Kasprzak H, Podbileska H, von Bally G, Fechner G. Biomechanical investigation of the hyoid bone using speckle interferometry. Int J Leg Med. I993;106:132-134.

3. Image - J, versión I.37 para Windows. http://rsb.info.nih.gov/ij/ 4. Papadopoulos N, Lykaki-Anastopoulou G, Alvanidou EL. The shape and size of the human hyoid bone and proposal for an alternative classification. J Anat.1989;163:249-260. http://www.pubmedcentral.nih.gov/picrender.fcgi?artid $=1256534 \&$ blobtype $=$ pdf.

5. Williams PL, Bannister LH, Berry MM, Collins P, Dyson M, Duseck JE, Ferguson MWJ. Tratado de Anatomía de Gray. Versión online. http://www.bartleby.com/l07.

6. Black SM, Scheuer JL. Developmental Juvenile Osteology. Academic Press. 2000.

7. Freyschmidt J, Brossmann J, Wiens J, Sternberg A. Borderlands of normal and early pathological findings in skeletal radiography. 5th English Edition, 2.00I. George Thieme Verlag.

8. Omnell KAH, Gandhi C, Omnell L. Ossification of the human stylohyoid ligament. Oral Surg Oral Med Oral Pathol Oral Radiol Endod. 1998;85:226-32.

9. 0 'Halloran RL, Lundy JK. Age ans ossification of the hyoid bone: forensic implications. J Forensic Sci.1987;32(6):1655-1659.

10. Miller KWP, Walker PL, $0^{\prime}$ Halloran RL. Age and sex-related variation in hyoid bone morphology. J Forensic Sci.1998;43(6):I1381143.

II. Casas JD, Rodríguez MS. Manual de Medicina Legal y Forense. Colex.2000.

I2. Di Maio DJ, Di Maio VJM. Forensic Pathology. CRC Press. 1993.

13. Knight B. Simpson's Forensic Medicine. I0th Edition. Arnold. 1991.

14. Gisbert JA. Medicina Legal y Toxicología. 5a Edición. Masson.1998.

15. Knight B. Forensic Pathology. 2nd Edition. Arnold. 1996.

16. Saukko P, Knight B. Knight's Forensic Pathology. 3rd Edition. Arnold. 2004.

17. Di Maio VJM. Homicidal asphyxia. Am J Forensic Med Pathol.2000;21(I):I-4.

18. Sánchez de León MS. Hallazgos microscópicos en la autopsia del cuello: casuistica del Instituto de Toxicología en casos de ahorcadura, estrangulación y otras lesiones cervicales. Cuad Med For.2001;23:31-47.

19. Felgin $\mathrm{G}$. Frequency of neck organ fractures in hanging. Am J Forensic Med Pathol.1999;20(2):128-130.

20. Betz P, Eisenmenger W. Frequency of throat-skeleton fractures in hanging. Am J Forensic Med Pathol.1996;17(3):191-193.

21. Maxeiner H, Ehrlich E, Schyma C. Neck injuries caused by being run over by a motor vehicle. J Forensic Sci.2000;45(I):3I-35.

22. Szeremeta W, Morovati SS. Isolated hyoid bone fracture: a case report and review of the literature. J Trauma.1991;31(2):268-271.

23. Raven KP, Reay DT, Harruff RC. Artifactual injuries of the Larynx produced by resucitative intubation. Am J Forensic Med Pathol.1999;20(I):31-36.

24. Gregersen M, Vesterby A. latrogenic fractures of the thyroid bone and the thyroid cartilage: a case report. Forensic Sci Int. 1981;17:41-43 25. Gordon I, Shapiro HA, Taljaard JJF, Engelbrecht HE. Aspects of the hyoid-larynx complex in forensic pathology. Forensic Sci.1976; $7: 161-170$.

26. Eliachar I, Goldsher M, Golz A, Joachims HZ. Hyoid bone fracture with pharyngeal lacerations. J Laryngol Otol.1980;94(3):331-5.

27. Olu lbekwe A. Fracture of the hyoid bone with lacerations of the pharynx. West Afr J Med.1991;10(2):198-200.

28. Anthony R, Martin-Hirsch D, England J. Dysphagia secondary to iatrogenic hyoid bone fracture. Br J Neurosurg.2000;14(4):337-8.

29. Kaufman HJ, Ciraulo DL, Burns RP. Traumatic fracture of the hyoid bone: three case presentations of cardiorespiratory compromise secondary to missed diagnosis. Am Surg. I999;65(9):877880.

30. Maxeiner H. Healed fractures of the larynx and lingual bone in forensic autopsy. Arch Kriminol.1999;203(5-6):175-183.

31. Khokhlov VD. Knitted fractures of the laryngopharynx framework as a medico-legal matter. Forensic Sci Int.1999;104:147-162.

32. Koebke J, Saternus KS. Zür morphologie des adulten menschlichen Zungenbeis. Zeitschrift für Rechtsmedizin. 1979;84:7-18.

33. Harjeet, Jit I. Shape, size and secular dimorphism of the hyoid bone in Northwest indians. J Anat Soc India. 1996;45(I):4-22. 
34. Pollanen MS, Ubelaker D. Forensic significance of the Polymorphism of hyoid bone shape. J Forensic Sci.1997;42(5):890892.

35. Pollanen MS, Bulger B, Chiasson DA. The location of hyoid fractures in strangulation revealed by Xeroradiography. J Forensic Sci.1995;40(2):303-305.

36. Pollanen MS, Chiasson DA. Fracture of the hyoid bone in strangulation: comparison of fractured and unfractured hyoids from victims of strangulation. J Forensic Sci.1996;4I(I):II0-II3.
37. Di Nunno N, Lombardo S, Costantinides F, Di Nunno C. Anomalies and alterations of the hyoid-larynx complex in forensic radiographic studies. Am J Forensic Med Pathol.2004;25(I):14-19.

38. Khokhlov VD. Injuries to the hyoid bone and laryngeal cartilages: effectiveness of different methods of medico-legal investigation. Forensic Sci Int.1997;88:173-183.

39. Di Nunno N, Costantinides F, Bernasconi P, Lombardo S. Radiographic magnification in the diagnosis of traumatic lesions of the hyoid-larynx complex. Int J Leg Med. 1998;III:38-42. 